\title{
EESTI KEELE PEEGELKEEL - AALPU TAASA KAADASTAAS
}

Siin on tuntud laul, korrapäraselt ümber vahetatud kirjatähtedega. Pealkirjas antud „tõlge” annab enam kui piisava võtme laulu tabamiseks ja see omakorda täielikuks muunduste tabeliks. See eesti keele peegelkeel sisaldab samu tähti, ulatuslikult samade esinemisnormidega silbilõpus ja tagasilpides. Seetõttu keele rütm ja põhiline kõla säilib enam-vähem. Erinevusi keele kõlas põhjustab eeskätt ümbervahetatavate tähtede erinev esinemissageduse muster. Raskesti hääldatavaid häälikuühendeid juhtub, ent vähe.

\section{Nepl esepu õm pigsu naal, au tabedu pe kasde. \\ Au tinnevbe pe letle aal, au ponge täävi lasde. \\ Tõvb elpil õne kengide \\ Nepl sögu noule oia. \\ Lekl pisu relpi tövtubal \\ tiu touda tivhan toia.}

\section{Tel lume pogvel kevimup}

au amen pimbe peje?

Rou õm lis nimep näplu laal, ap näpl au rope neje?

Nepl ropeg näplu neje täss, luul lauleg laa pes koial.

Laa lämmug põel, tuvutil, tiub nuppa noule oial.

Ja tõesti:

He tiu laa seis naus tõvbe söag, au naua tunki höö!

Kuigi laulu varal saab koostada täieliku muunduste tabeli, annan selle siin ka otseselt. Järgnevalt seletan, miks just need valisin ja millised raskused püsivad.

$$
\begin{array}{lrl}
a \leftrightarrow e & \ddot{a} \rightarrow \ddot{o} \rightarrow & \ddot{u} \rightarrow \ddot{a} \\
i \leftrightarrow u & o \leftrightarrow \tilde{o} \\
k \rightarrow t \rightarrow p \rightarrow k & l \leftrightarrow s \\
g \rightarrow d \rightarrow b \rightarrow g & h \leftrightarrow j \\
\eta \rightarrow n \rightarrow m \rightarrow \eta & r \leftrightarrow v
\end{array}
$$$$
\text { (kus nasaalne } \eta \text { ilmneb } n \text {-ina) }
$$

Omavahel peab muundama neid täishäälikuid, mis tohivad esineda ainult sõna esimeses silbis. Keelekõla ja sageduse poolest on mõtet omavahel muundada tagahäälikud $(o \leftrightarrow \tilde{o})$ ja roteerida kolm eeshäälikut $(\ddot{a} \rightarrow \ddot{o} \rightarrow \ddot{u} \rightarrow \ddot{a})$. Sama hästi (või halvasti) võiks roteerida ka vastassuunas $(\ddot{a} \leftarrow \ddot{o} \leftarrow \ddot{u} \leftarrow \ddot{a})$.

Võiks arvata, et keelekõla mõttes oleks soovitav omavahel muundada teised tagahäälikud ( $a \leftrightarrow u$ ) ja samuti eeshäälikud $(e \leftrightarrow i)$. Siis aga saaksime ebatavalised kaksikhäälikud (ei $\rightarrow$ ie ja $a u \rightarrow u a)$. On sujuvam muundada $a \leftrightarrow e$ ja $i \leftrightarrow \mathrm{u}$, nii et $e i \leftrightarrow a u$. (Foneetiliselt on see pigem $e j \leftrightarrow a w$, aga ma püsin kirjakeele raames.) Kõik $a, e, i, u$ omavahelised kaksikhäälikud muunduvad hääldatavalt.

Naastes teiste täishäälikute juurde neid sisaldavaist diftongidest kolmandik muundub hääldatavaks (oa $\leftrightarrow \tilde{o} e$ ), kolmandik raskesti hääldatavaks $(\ddot{a} i \rightarrow \ddot{o} u)$ ja kolmandik lausa võimatuks (öi $\rightarrow \ddot{u} u$ ). Need viimased esinevad õnneks harva.

Kaashäälikute osas on ilmselt vaja roteerida kas $k \rightarrow t \rightarrow p \rightarrow k$ või $k \rightarrow p$ $\rightarrow t \rightarrow k$. Esimese eelis on see, et ülisage $k$ muundub poolsagedaks $t$-ks pigem kui haruldasemaks $p$-ks. Sellest tuleneb siis paratamatult ka $g \rightarrow d \rightarrow b \rightarrow g$ ja $\eta \rightarrow n$ $\rightarrow m \rightarrow \eta$. Viimane jada on ilmsem, kui mõelda ühendeile $\eta g \rightarrow n d \rightarrow m b \rightarrow \eta g$ ja $\eta k \rightarrow n t \rightarrow m p \rightarrow \eta k$. Eesti kirjaviis paraku peidab selle $\eta$-i ära, asendades ta $n$-iga. Tulemuseks on siis, et $n g-i$ just nagu üldse ei muunduks. 
Kuna $j$ ja $h$ esinevad valdavalt silbi alguses, on mõtet neid paari panna: $j \leftrightarrow h$. Harvad silbilõpu $h$-d toodavad siis $j$-i sõna lõpus - kirjaviisi vastaselt, ehkki mitte hääldamise vastaselt, sest eesti $e i$ ongi pigem $e j$, erinevalt soome $e i$-st.

Jäävad järele $l, r, s$ ja $v$. Tundub loomulikuna panna paari $l$ ja $r$, ja $l \leftrightarrow r$ toimikski üsna sujuvalt. Aga siis jääks järele vaid karvu turri ajav $s \leftrightarrow v$. Üks on helitu, teine heliline, ent veel raskem on see, et $s$ on sage, tihti ka sõna lõpus, kuna $v$ esineb harvemini, eriti veel sõna lõpus. Mõttesse tuleb siis hoopis $s \leftrightarrow l$, mis vahetab ilusasti ümber seesütleva ja alalütleva sõnalõpud. Seetõttu mõjub sage $l$ sõna lõpus loomulikumalt kui sage $v$.

Sel puhul jääb üle vaid muundada $r \leftrightarrow v$, mis ei tee enamasti raskusi. Ainultjärv $\rightarrow$ hövr toob sisse natuke saami hõngu.

Kogusummas on üllatav, millises ulatuses tavalised häälikuühendid annavad talutava peegelvaste. Haruldasematega esineb õudsusi, eriti $o$-lõpulistel diftongidel, nagu käo $\rightarrow$ töõ ja lao $\rightarrow$ seõ.

Harva juhtub, et peegelvastel on tähendus tavakeeles. Antud laulus leidub neid vaid neli: au, lume, luul ja seis. See näidistab, kui väheselt kasutab keel ära ka hääldatavaid häälikuühendeid, sellal kui mõned ühendid on üle koormatud mitmete tähendustega. Terve lause peegeldub poolmõttekalt vaid harva: Kisad seal veel, sina, kiisk $\rightarrow$ Tuleb laes raas lumetuult.

Tähendusega vasteid esineb teadagi enim lühikestes sõnades. Kui nad ei sisalda täpiga ega $k$-t-p( $\eta g-n d-m b)$-tähti, on muude mõlemasuunaline, tõeline peegel: $a u \leftrightarrow e i, \tilde{o} e \leftrightarrow o a$, ala $\leftrightarrow e s e$ õle $\leftrightarrow$ osa, rehe $\leftrightarrow$ vaja, vaas $\leftrightarrow$ reel, lill $\leftrightarrow$ suss, seisa $\leftrightarrow$ laule, laulis $\leftrightarrow$ seisul, sealaes $\leftrightarrow$ laeseal. Muudel puhkudel on muude ühesuunaline, nii et tagasipööret pole ja iseloomustus peegelkeelena on tinglik: sang $\rightarrow$ lend, ling $\rightarrow$ sund, laat $\rightarrow$ seep, kava $\rightarrow$ tere, laama $\rightarrow$ seene, $l i$ sad $\rightarrow$ suleb, rajud $\rightarrow$ vehib.

Enam kui 5-tähelistel lihtsõnadel kohtab tähendusega muudet harva, kuigi leidub sooned $\rightarrow$ lõ̃mab ja isegi 7-täheline tabelil $\rightarrow$ pegasus. Liitsõnadest on ehk rekordi lähedal purukivinööp $\rightarrow$ kiviturumüük.

Muudet üha korrates jõuab muidugi tagasi algusse pärast 6 permutatsiooni, millest enamus annab tähenduseta sõnad. Harva juhtub, et kõigil kuuel sõnal on tähendus: pikk $\rightarrow$ kutt $\rightarrow$ tipp $\rightarrow$ pukk $\rightarrow$ kitt $\rightarrow$ tupp $\rightarrow$ pikk..., paas $\rightarrow$ keel $\rightarrow$ taas $\rightarrow$ peel $\rightarrow$ kaas $\rightarrow$ teel $\rightarrow$ paas... ja paraku ka pill $\rightarrow$ kuss $\rightarrow$ till $\rightarrow$ puss $\rightarrow$ kill $\rightarrow$ tuss $\rightarrow$ pill... (vähemalt ÕS 1976-s esineb kill omaette sõnana).

Võib-olla aitab see ulakas mäng paremini tajuda eesti keele häälikusuhteid ja nende väljendust kirjas. Ma ei tea, kui hästi saaks teha säärast mängu teistes Euroopa keeltes. Lihtsam võiks see olla polüneesia keeltes, kus erinevaid häälikuid on vähe, ja võib-olla ühesilbilistes keeltes. Ülimalt ebafoneetilised kirjakeeled on vist lootusetud. 


\section{Peegelkeele teine murre}

Igaks juhuks olgu siin antud ka näide sellest, mis juhtub, kui valida hoopis rotatsioon $k \rightarrow p \rightarrow t \rightarrow k$ ja muundused $s \leftrightarrow v$ ning $l \leftrightarrow r$. Täishäälikute muuted ja $j \leftrightarrow h$ jäävad samaks. Häälikuliselt $m \rightarrow n \rightarrow \eta$ tooks siis sisse $\eta$-i eraldi $\eta g$ st või $\eta k$-st, mis on omal kohal samojeedi keeltes, aga mitte läänemeresoome keeltes. Eesti kirjaviis peidaks küll selle $\eta$-i ära, asendades ta $n$-iga. Siis aga paistaks, et $n$ just nagu üldse ei muunduks: tuleb $n \leftrightarrow n$ iga tavalise $n$-i puhul.

$$
\begin{array}{lr}
a \leftrightarrow e & \ddot{a} \rightarrow \ddot{o} \rightarrow \ddot{u} \rightarrow \ddot{a} \\
i \leftrightarrow u & o \leftrightarrow \tilde{o} \\
k \rightarrow p \rightarrow t \rightarrow k & l \leftrightarrow r \\
g \rightarrow b \rightarrow d \rightarrow g & j \leftrightarrow h \\
\eta \rightarrow m \rightarrow n \rightarrow \eta & s \leftrightarrow v \\
\text { (kus } \eta \text { ilmneb } n \text {-ina) } &
\end{array}
$$

Raskemini hääldatavaid vorme tuleb selles murdes rohkem. Ja isegi kui sõnalõpu $v$ on vabalt hääldatav, mõjub ta sagedus seal imelikult. Tähendusega muunduvaid sõnu on laulus ainult üks (au).
Nekv ereku õn kidru naav, au pagebu ke tarbe.

Au pinnelge ke vepve aav, au konde tääli varbe.

Põlg evkiv õne tendibe Nekv rödu nouve oia. Vepv kiru sevki pölpugav piu pouba pilhan poia.

Pev vune kodlev telinuk au anen kinge keje?

Sou õn vir nineg näkvu vaav, ak näkv au soke neje?

Nekv soked näkvu neje pärr, vuuv vauved vaa ker toiav. Vaa vännud kõev, pulupiv, piug nukka nouve oiav.

He piu vaa reir naur põlge röad, au naua punti höo!

REIN TAAGEPERA 